\title{
A supernilpotent non-special radical class
}

\section{L.C.A. van Leeuwen and T.L. Jenkins}

\author{
Let $F$ be the upper radical determined by all fields. The \\ supernilpotent radical classes which are not special have thus \\ far always contained $F$ properly. The purpose of this note is \\ to construct a countably infinite number of supernilpotent rad- \\ ical classes which are not special and each of which is properly \\ contained in $F$. The construction involves a ring due to \\ Leavitt which is interesting in its own right and is not \\ generally known. All rings considered are associative.
}

1.

Let $F$ be the upper radical determined by all fields. The supernilpotent radical classes which are not special (see [2] and [3]) have thus far always contained $F$ properly. The purpose of this note is to construct a countably infinite number of supernilpotent radical classes which are not special and each of which is properly contained in $F$. The construction involves a ring due to Leavitt which is interesting in its own right and is not generally known. All rings considered will be associative and standard radical theory terminology can be found in $[1]$.

If $M$ is a hereditary class of rings we will let $U M$ denote the upper radical class determined by $M$ and SUM the corresponding semisimple class. Recall that a ring $A$ is in SUM if and only if every nonzero ideal of $A$ has a nonzero homomorphic image in $M$. In addition, $B$ and $G$ will denote the lower Baer and Brown-McCoy radicals respectively.

Received 4 June 1973. 
Let $U M$ be a supernilpotent radical class determined by the special class $M$. There are several conditions on $U M$ which are known to be equivalent to the property that $U M$ is a special supernilpotent radical class. We will need the following equivalence.

LEMMA 1. If UM is a supernilpotent radical class then UM is special if and only if in every UM-semisimple ring $A$ there exists $a$ proper prime ideal $P$ such that $A / P$ is uls-semisimple.

Proof. If $U M$ is a special radical class then every $U M$-semisimple ring $A$ is isomorphic to a subdirect sum of rings from the special class $M$ [1, Lemma 80]. Each nonzero summand is of the form. $A / P$ where $P$ is a proper prime ideal of $A$. Conversely, let $N$ be the class of all prime $U M$-semisimple rings. Since $U M$ is supernilpotent, $N$ is a special class [1, Lemma 85] and by definition of an upper radical $U M \subseteq U N$ and hence $S U N \subseteq S U M$. Now let $A \in S U M$ and $I$ be a nonzero ideal of $A$. Then $I \in S U M$ and by our assumption $I$ has a nonzero prime UM-semisimple image. Thus, by definition $A \in S U N$. Hence $S U N=S U M$ and $U M$ is a special radical class.

DEFINITION. A class $W$ is a weakly special class of rings if it satisfies the following three conditions:

(1) every ring in the class $W$ is semiprime;

(2) $W$ is hereditary;

(3) if $A \in W$ and $A$ is an ideal in a ring $K$ where $A^{*}=0$, then $K \in W$.

Here, $A^{*}$ is the annihilator of $A$ in $K$.

In [2], Rjabuhin shows that any upper radical determined by a weakly special class is supernilpotent. He then constructs a supernilpotent nonspecial radical class containing $F$ properly. Snider [3] constructs another such class.

2.

With the aid of a ring due to Leavitt we are able to construct a supernilpotent radical class which is not special yet is contained properly in $F$. We first give the properties of this ring, call it $A$, which are 
best described by a diagram as follows:
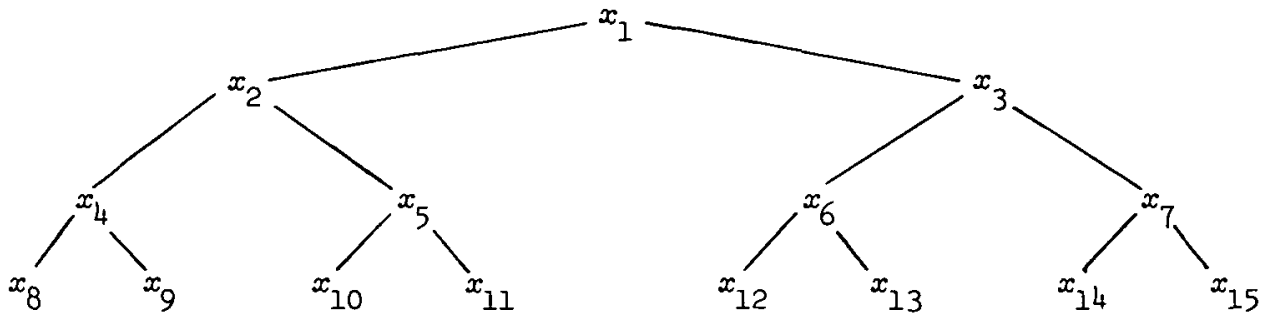

The ring $A$ is to be generated over $z_{2}$, the field of order 2 , by the countably infinite set of symbols $\left\{x_{i}\right\}$, with the following relations: any $x_{i}$ is the sum of the two immediately below (thus $x_{2}=x_{4}+x_{5}$ ); any $x_{i}$ is the identity for all those $x_{j}$ equal to or below it but has zero product with incomparable $x_{j}$ (thus $x_{2} x_{9}=x_{9} x_{2}=x_{9}$ but $\left.x_{2} x_{7}=x_{7} x_{2}=0\right)$. By $\left(x_{i}\right)$, below, we mean the set of $x_{j}$ which are equal to $x_{i}$ or below and comparable to $x_{i}$. Some of the properties of the ring $A$ are:

(a) $A=\left(x_{1}\right)$ is isomorphic to the principal ideals, such as $\left(x_{5}\right)$;

(b) every ideal of $A$ is the direct sum of such principal ideals;

(c) the only prime image of $A$ is $Z_{2}$ which you would get, say, by $A / I$. where $I=\left(x_{2}, x_{6}, x_{14}, \ldots\right)$;

(d) the only prime ideals of $A$ are maximal ideals;

(e) $A$ has no nonzero nilpotent ideals and so is B-semisimple;

(f) the intersection of all maximal ideals is zero;

(g) $A$ is a boolean ring with the identity $x_{1}$;

(h) A does not contain $Z_{2}$ as an ideal, for every ideal of $A$ is countably infinite.

THEOREM 1. Let $S$ be a simple ring with unit which is not a field. Let $T$ be a class of rings $A$ satisfying the following 
conditions :

(a) A is a subdirect ston of copies of $S$;

(b) $S$ is not an ideal in $A$.

Let $F$ be the class of all fields. Then if $M=F \cup T, M$ is a weakly special class.

Proof. Every ring in $M$ is G-semisimple and hence B-semisimple and thus semiprime. To show that $M$ is hereditary let $a \in M$ and $I \neq 0$ be an ideal of $A$. Since $A$ is a subdirect sum of copies of $S, A$ contains a class of maximal ideals $\left\{M_{i}\right\}$ such that $n M_{i}=0$ and $A / M_{i} \cong S$ for each $i$. Hence $I \cap M_{i}$ is a prime ideal in $I$ for each $i$ and $\cap\left(I \cap M_{i}\right)=0$. Thus $I$ is a subdirect sum of rings $I /\left(I \cap M_{i}\right) \cong\left(M_{i}+I\right) / M_{i}$. It is clear that $I \$ M_{i}$ for at least one $i$ and in this event $\left(M_{i}+I\right) / M_{i} \cong A / M_{i} \cong S$. On the other hand, if $I \subseteq M_{i}$ then $\left(M_{i}+I\right) / M_{i}=0$. Hence $I$ is a subdirect sum of copies of $S$. If $S$ was an ideal in $I$ then $0 \neq\left(S^{\prime}\right)^{3} \subset S \subset S^{\prime} \subset I \subset A$ where $S^{\prime}$ denotes the ideal of $A$ generated by $S$. But $S$ is simple so $\left(S^{\prime}\right)^{3}=S$ and $S$ would be an idf of $A$. Hence $M$ is hereditary.

Now let $B \in M$ where $B$ is an ideal in the ring $A$ with $B^{*}=0$. If $B, \in F$ then $A=B+C$ and $C \subset B^{*}=0$. Hence $A=B \in M$. Now suppose $B \in T$. Then $B$ contains a class of maximal prime ideals $\left\{P_{i}\right\}$ with $\cap P_{i}=0$ and $B / P_{i} \cong S$ for each $i$. Define for each $P_{i}$, $P_{i}^{*}=\left\{x \in A \mid B x \subseteq P_{i}\right\}$. Then $P_{i}^{*}$ is an ideal in $A$ and we claim that $P_{i}=P_{i}^{*} \cap B$ for each $i$. It is clear that $P_{i} \subseteq P_{i}^{*} \cap B$ so let $b \in P_{i}^{*} \cap B$. Then $B b \subseteq P_{i}$, and hence $B(b)_{r} \subseteq P_{i}$, where $(b)_{r}$ denotes the right ideal of $B$ generated by $b$. Therefore $(b)_{r}^{2} \subseteq P_{i}$, which implies $(b)_{p} \subseteq P_{i}$, for $P_{i}$ is prime in $B$, and hence $b \in P_{i}$. Now we show $A=P_{i}^{*}+B$ for each $i$. Let $e+P_{i}$ be the identity in $B / P_{i}$, $e \in B$. Then $y e=y\left(P_{i}\right)$ for any $y \in B$, so yex $=y x\left(P_{i}\right)$ for any 
$y \in B$ and any $x \in A$. But then $y(x-e x)=y x-y e x \equiv y x-y x\left(P_{i}\right)$, so $y(x-e x) \in P_{i}$ for any $y \in B, x \in A$. This implies that $x-e x \in P_{i}^{*}$ for any $x \in A$. From $x=(x-e x)+e x$ with $x-e x \in P_{i}^{*}$ and $e x \in B$ it follows that $A=P_{i}^{*}+B$. Hence $A / P_{i}^{*}=\left(B+P_{i}^{*}\right) / P_{i}^{*} \cong B /\left(B \cap P_{i}^{*}\right)=B / P_{i} \cong S$. Also $n P_{i}=B \cap\left(n P_{i}^{*}\right)=0$ which says $n P_{i}^{*} \subseteq B^{*}=0$, so $n P_{i}^{*}=0$. Hence $A$ is a subdirect sum of copies of $S$.

Finally, suppose that $S$ is an ideal in $A$. Since $B \in M, S$ is not an ideal of $B$ so $S \cap B=0$. Thus $S B=B S=0$ or $S \subseteq B^{*}=0$, a contradiction. Hence $S$ is not an ideal of $A$ and $A \in T \subset M$ completing the proof.

By choosing an appropriate simple ring with unit we show in the following lemma that the class $T$ of the preceding theorem is non-empty. It is here that the ring of Leavitt is used. The matrix theory used in the proof is an elementary consequence of using a ring with unit.

LEMMA 2. Let $S=\left(z_{2}\right)_{n}$, the ring of $n \times n$ matrices over the field $z_{2}$. The class $T$ of rings which are subdirect sums of copies of $S$ and which do not contain $S$ as an ideal is non-empty.

Proof. The ring $S$ is a simple ring with unit but not a field. Where $A$ denotes Leavitt's ring, we show that $A_{n} \in T$. The ring $A$ has a unit, so every ideal in $A_{n}$ has the form $I_{n}$ where $I$ is an ideal in $A$. First we show that if $I_{n}$ is a prime ideal in $A_{n}$, then $A_{n} / I_{n} \cong S$. Since $A_{n} / I_{n} \cong(A / I)_{n}$ it follows that $(A / I)_{n}$ is a prime ring and hence $A / I$ is a prime ring. The only prime image of $A$ is $z_{2}$ so $A / I \cong Z_{2}$ and $A_{n} / I_{n} \cong(A / I)_{n} \cong\left(z_{2}\right)_{n}=S$. This also shows that every prime ideal in $A_{n}$ is maximal.

Since $B(A)=0, B\left(A_{n}\right)=(B(A))_{n}=0$. Hence $A_{n}$ is a subdirect sum of prime rings, that is, there exists a class $\left\{\left(P_{i}\right)_{n}\right\}$ of prime ideals in $A_{n}$ with $\cap\left(P_{i}\right)_{n}=0$. From the above we get $A_{n} /\left(P_{i}\right)_{n} \cong S$ for any $\left(P_{i}\right)_{n}$. Hence $A_{n}$ is a subdirect sum of copies of $S$. As $Z_{2}$ is not an 
ideal in $A,\left(Z_{2}\right)_{n}=S$ is not an ideal in $A_{n}$. Hence $A_{n} \in T$ and $T$ is non-empty.

The class $M=T \cup F$ is a weakly special class and hence $U M$ is supernilpotent [2]. We show that $U M$ is not special by contradicting the equivalence of Lemma 1 .

THEOREM 2. Let $T$ be the class of all subdirect sums of copies of $\left(z_{2}\right)_{n}$ not containing $\left(z_{2}\right)_{n}$ as an ideal and let $F$ be the class of all fields. Then the upper radical class UM determined by $M=T \cup F$ is supermilpotent, not special, and $U M \subset F$.

Proof. There only remains to show that $U M$ is not special. $A_{n} \in T$ so $A_{n}$ is UM-semisimple. The only prime image of $A_{n}$ is $\left(z_{2}\right)_{n}$. Since $\left(z_{2}\right)_{n}$ is simple and not in $M,\left(z_{2}\right)_{n}$ must be $U M$-radical. Hence for $A_{n}$ there does not exist a proper prime ideal $P_{n}$ such that $A_{n} / P_{n}$ is UM-semisimple and hence $U M$ is not special by Lemma 1 .

\section{References}

[1] Nathan Divinsky, Rings and radicals (University of Toronto Press, Toronto, 1965).

[2] Ю.1\%. Рябухин [Ju.M. Rjabuhin], О наднильпотентных и спецнальных радиналах [On hypernilpotent and special radicals]. Issled. po algebre i matem. conalizu, 65-72 ("Kartja Moldovenjaske", Kišinev, 1965). Translated by William G. Leavitt (unpublished).

[3] Robert L. Snlder, "Lattices of radicals", Pacific J. Math. 40 (1972), 207-220.

Mathematisch Instituut,

Rijksuniversiteit te Groningen,

Groningen,

Netherlands;
Department of Mathematics,

University of Wyoming,

Laramie,

Wyoming,

USA. 